\title{
Inadequate Cooling Identified
}

National Cancer Institute

\section{Source}

National Cancer Institute. Inadequate Cooling Identified. NCI Thesaurus. Code C139519.

The device did not sufficiently cool the patient or another device during operation. 